\title{
Testing Models Relating Rejection, Depression, Interpersonal Needs, and Psychache to Suicide Risk in Nonclinical Individuals
}

\author{
Rui C. Campos ${ }^{1}$ and Ronald R. Holden ${ }^{2}$ \\ ${ }^{1}$ University of Évora, Portugal \\ ${ }^{2}$ Queen's University, Canada
}

Objectives: Using structural equation modeling, we tested a primary model of suicide risk and 3 competing, alternative models based on 4 psychological variables deemed important in the literature (perception of parental rejection, depression, interpersonal needs comprising perceived burdensomeness and thwarted belongingness, and psychache), in a nonclinical sample of Portuguese adults. Method: A convenience sample of 203 adults ( 100 men, 103 women; aged18-65 years) participated in this study. Results: Analyses demonstrated that the proposed primary model had the best fit to the observed data. The differences in fit indexes for this model and one of the alternative models, however, were not substantial. Conclusion: Perceived parental rejection related directly to suicide risk and indirectly via depression and interpersonal needs. Depression linked indirectly to suicide risk via interpersonal needs and psychache. Interpersonal needs related directly to suicide risk and indirectly via psychache, which related directly to suicide risk. (C) 2015 Wiley Periodicals, Inc. J. Clin. Psychol. 00:1-10, 2015.

Keywords: rejection; depression; psychache; interpersonal needs; suicide risk; community-based sample

Suicide is a major health problem across the lifespan for both clinical and nonclinical populations. Although suicide is a low base-rate phenomenon, it is an important mental health issue (World Health Organization, 2012), and, as such, the identification of relevant factors associated with suicide risk is a critical research endeavour. In Portugal, over 1,000 people die by suicide every year (10.3 per 100,000 in the population; National Institute of Statistics [NIS], 2013) and suicide attempts are estimated to have a rate 10 to 20 times higher than deaths by suicide (Fotti, Katz, Afifi, \& Cox, 2006).

Furthermore, suicidality is not only an issue for Portugal but also a significant public health concern worldwide. In Portugal, suicide is a demographic-based phenomenon, with higher rates in the southern part of the country, the Alentejo region (NIS, 2013). It is not completely clear why this regional difference (endemic phenomenon in Alentejo) occurs, but a lower level of religiosity compared with the northern part of the country may be one possible explanation. Although it is recognized that suicide takes place within a specific cultural context, in the present study, we focus on psychological variables, rather than sociocultural factors. As such, our study may be of an etic nature.

The prediction of suicide remains extremely difficult (Overholser, Braden, \& Dieter, 2012) because suicide behaviors are regarded as multifactorial phenomena (e.g., Hawton \& van Heeringe, 2009). Psychological perspectives of suicide (e.g., Goldney \& Schioldann, 2004) have demonstrated the existence of potential interactions among various individual psychological risk factors (e.g., Beautrais, Collings, Ehrhardt, \& Henare, 2005). In particular, several psychological variables that have been empirically related to suicide risk include the perception of parental rejection (e.g., Campos, Besser, \& Blatt, 2013), Shneidman's (1993) concept of psychache (e.g., Troister

We extend a grateful thanks to all the participants in this study and to the research assistants at the University of Évora, who collected the data.

Please address correspondence to: Rui C. Campos, Departamento de Psicologia, Escola de Ciências Sociais, Universidade de Évora, Apartado 94, Évora, Portugal 7002-554. E-mail: rcampos@uevora.pt 